\title{
Revealing Early Stage Nucleation Events of Pharmaceutical Crystals Using Liquid Phase Electron Microscopy
}

Jennifer Cookman ${ }^{1}$, Victoria Hamilton ${ }^{2}$, Simon Hall ${ }^{2}$ and Ursel Bangert ${ }^{1}$

${ }^{1}$ University of Limerick, Limerick, Limerick, Ireland, ${ }^{2}$ University of Bristol, Bristol, England, United Kingdom

Liquid phase electron microscopy has enabled the direct observation of liquid phase events that had previously been unexplored in situ at the nanoscale such as nanoparticle nucleation, electrochemical dynamics, catalysis transformations.[1, 2, 3] So far the information gathered utilising this invaluable in situ technique has gathered traction for inorganic materials as well as soft materials owing to the performance of instrumentation paired with in situ equipment e.g. TEM environmental holders and direct electron detectors.

The materials focussed on are Active Pharmaceutical Ingredients (APIs), which are vital products in pharmaceutical drugs and contain molecules oriented in a specific crystal structure (polymorph) that determine its bioavailability and solubility governing its effectiveness inside the body. Understanding how the dominant crystal structure manifests over less stable crystal structures is vital, not only for the pharmaceutical industry, but also for understanding the early stages of the crystal growth i.e. nucleation.

Here, we show early stage events of the liquid phase growth of a NonSteroidal Anti-Inflammatory Drug (NSAID), flufenamic acid (FFA). By using a variety of concentrations of the material in a compatible solvent, different observations have been recorded suggestive of Pre-Nucleation Clusters (PNCs) at low concentrations and hexagonal crystals characteristic of the crystals structure of the most stable form of FFA.

Notable visualised events are compared to descriptions and current recordings of existing nucleation theories following on from previous work by the authors that revealed crystal growth of the same material in undersaturated conditions. [4] The notable density fluctuation that is described in multi step nucleation theories is visualised in suggested PNC's where liquid-like formation contributes to the formation of the new, electron dense crystalline entity (figure 1). A similar density fluctuation is also recorded at higher concentrations after the formation of hexagonal crystals where the crystals appears to act as seeding point to nucleate additional crystalline particles (figure 2).

Vital considerations of the liquid phase experiments include radiolysis and how the unavoidable radiolysis products interact with the molecules in solution. For the crystal nucleation to occur it is hypothesis that radiolysis is vital in causing molecular assembly to generate the unit cell and hence the nuclei for further crystal growth.

Other aspects of liquid phase electron microscopy experiments are discussed such as concentration, imaging mode, liquid thickness and electron dose. 

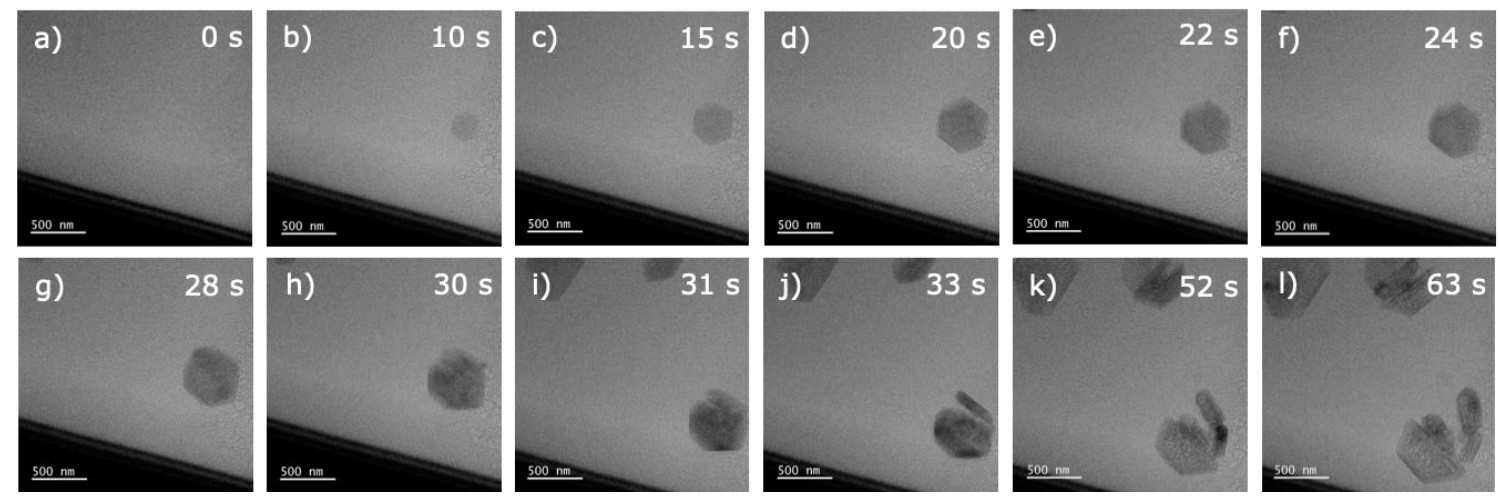

Figure 1. a) to d) A series of images following the formation of an FFA crystal. e) to $h$ ) the hexagonal crystal began to flicker with a dynamic change in electron density suggesting transformation of the structure. i) to 1) After 31 s the crystal began to split revealing some smaller crystals. Dose rate: $2 \mathrm{e}-/ \AA 2 / \mathrm{s}$.
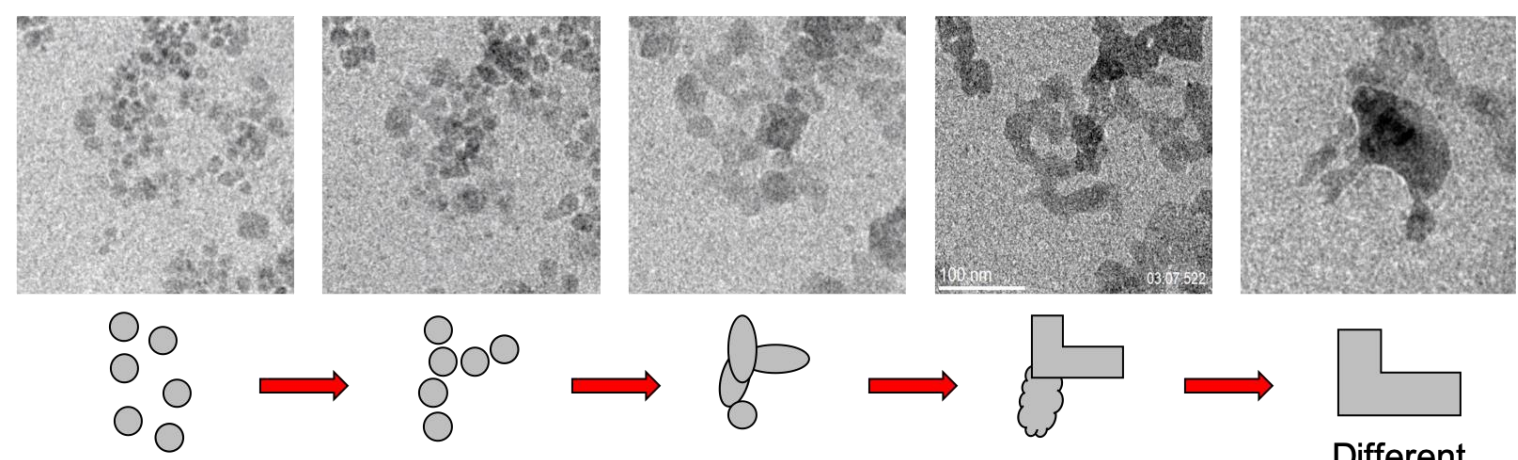

Individua

I clusters
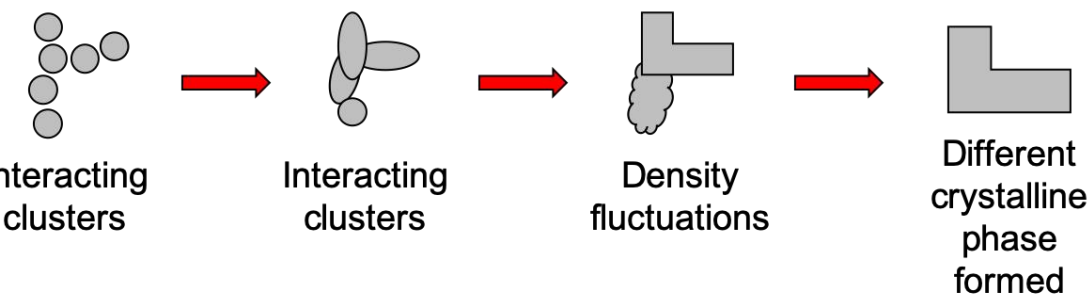

Figure 2. A comparison of the particular observed crystal growth event to "multi-step nucleation" with the most notable event being the density fluctuation and transformation from disordered liquid-like substance to an electron dense ordered, crystalline entity.

\section{References}

1.De Yoreo, J. J. (2016). In-situ liquid phase TEM observations of nucleation and growth processes. Progress in Crystal Growth and Characterization of Materials, 62(2), 69-88.

2. Rong, G., Zhang, X., Zhao, W., Qiu, Y., Liu, M., Ye, F., ... \& Duan, W. (2017). Liquid-Phase Electrochemical Scanning Electron Microscopy for In Situ Investigation of Lithium Dendrite Growth and Dissolution. Advanced Materials, 29(13), 1606187.

3. Zhu, G. Z., Prabhudev, S., Yang, J., Gabardo, C. M., Botton, G. A., \& Soleymani, L. (2014). In situ liquid cell TEM study of morphological evolution and degradation of Pt-Fe nanocatalysts during potential cycling. The Journal of Physical Chemistry C, 118(38), 22111-22119.

4. Cookman, J., Hamilton, V., Price, L. S., Hall, S. R., \& Bangert, U. (2020). Visualising early-stage liquid phase organic crystal growth via liquid cell electron microscopy. Nanoscale. 